\title{
Ferramentas para a organização do processo de trabalho na segurança do paciente
}

Tools to organize the work process in patient safety

Herramientas para organizar el proceso de trabajo en seguridad del paciente

\author{
Marielli Trevisan Jost ${ }^{1}$ (]) \\ Aline Branco² (1) \\ Bárbara Rodrigues Araujo $^{3}$ (C) \\ Karin Viegas $s^{4,5}$ (1) \\ Rita Catalina Aquino Caregnato 4,5 [C]
}

1. Santa Casa de Misericórdia de Porto Alegre. Porto Alegre, RS, Brasil.

2. Programa de Residência Multiprofissional em Saúde, Grupo Hospitalar Conceição. Porto Alegre, RS, Brasil.

3. Universidade Federal de Ciências da Saúde de Porto Alegre. Porto Alegre, RS, Brasil.

4. Universidade Federal de Ciências da Saúde de Porto Alegre, Departamento de Enfermagem. Porto Alegre, RS, Brasil.

5. Universidade Federal de Ciências da Saúde de Porto Alegre, Programa de Pós-Graduação em Enfermagem. Porto Alegre, RS, Brasil.
Autor correspondente:

Marielli Trevisan Jost

marielli.jost@gmail.com

Recebido em 06/06/2020.

Aprovado em 27/11/2020.

DOl:https://doi.org/10.1590/2177-9465-EAN-2020-0210

\section{RESUMO}

Objetivo: discutir acerca da utilização das ferramentas de Análise de Modo e Efeitos de Falha e sua aplicação na assistência à saúde. Método: trata-se de um artigo de reflexão visando à apresentação do formato próprio de aplicação de ambas as ferramentas seguida das suas diferenças de execução nos processos de trabalho. Resultados: ambos os modelos possuem a mesma finalidade, sendo direcionados para a detecção de falhas antes mesmo da sua manifestação, auxiliando diretamente na promoção da segurança. A análise do erro, com a participação das equipes e a geração de índices de falhas, repercute no planejamento e na implementação de ações práticas voltadas à segurança do paciente. Conclusão e implicações para a prática: embora semelhantes, existem, entre eles, distinções quanto à priorização das falhas para elencar ações práticas corretivas, principalmente no cálculo do Índice de Prioridade de Risco relacionado à gravidade, na probabilidade de ocorrência e na detecção das falhas. Ambas as ferramentas se mostram como importantes aliadas dos gestores de saúde para a detecção de falhas graves que colocam em risco a assistência livre de eventos adversos.

Palavras-chave: Segurança do paciente; Gestão em Saúde; Avaliação de Processos em Cuidados de Saúde; Análise do Modo e do Efeito de Falhas na Assistência à Saúde; Qualidade da Assistência à Saúde.

\section{ABSTRACT}

Objective: to discuss the use of Failure Mode and Effects Analysis tools and their application in health care. Method: this is a reflection article, aiming at presenting the proper application format for both tools, followed by their differences in execution in the work processes. Results: both models have the same purpose, being directed to the detection of failures even before their manifestation, directly assisting in the promotion of safety. The analysis of the error with the participation of the teams and the generation of failure rates has repercussions on the planning and implementation of practical actions aimed at patient safety. Conclusion and implications for the practice: although similar, there are distinctions regarding the prioritization of failures to list practical corrective actions, mainly in the calculation of the Risk Priority Index related to severity, probability of occurrence and failure detection. Both tools are shown to be important allies to health managers for the detection of serious failures that put care free from adverse events at risk.

Keywords: Patient Safety; Health Management; Process Assessment, Health Care; Healthcare Failure Mode and Effect Analysis; Quality of Health Care.

\section{RESUMEN}

Objetivo: discutir el uso de las herramientas de Análisis de Modos y Efectos de Falla y su aplicación en la atención médica. Método: este es un artículo de reflexión, con el objetivo de presentar el formato propio de aplicación adecuado para ambas herramientas, seguido de sus diferencias de ejecución en los procesos de trabajo. Resultados: ambos modelos tienen el mismo propósito, dirigidos a la detección de fallas incluso antes de su manifestación, ayudando directamente en la promoción de la seguridad. El análisis del error con la participación de los equipos y la generación de tasas de fracaso tiene repercusiones en la planificación e implementación de acciones prácticas dirigidas a la seguridad del paciente. Conclusión e implicaciones para la práctica: aunque son similares, existen distinciones con respecto a la priorización de fallas para enumerar acciones correctivas prácticas, principalmente en el cálculo del Índice de Prioridad de Riesgo relacionado con la gravedad, la probabilidad de ocurrencia y la detección de fallas. Se ha demostrado que ambas herramientas son aliadas importantes para los gerentes de salud para la detección de fallas graves que ponen en riesgo la atención libre de eventos adversos.

Palabras clave: Seguridad del Paciente; Gestión en Salud; Evaluación de Procesos, Atención de Salud; Análisis de Modo y Efecto de Fallas en la Atención de la Salud; Calidad de la Atención de Salud. 


\section{INTRODUÇÃO}

As organizações de saúde necessitam desenvolver uma cultura de segurança de modo que sua força de trabalho e seus processos sejam focados na melhoria dos cuidados prestados. ${ }^{1}$ A National Patient Safety Foundation (NPSF) destaca as práticas que melhoram a segurança do paciente, reduzindo a ocorrência de eventos adversos evitáveis. ${ }^{2,3}$ Assim, melhorar a cultura de segurança é um componente essencial da prevenção ou redução de erros e da melhoria geral da qualidade da assistência à saúde. ${ }^{4}$

A segurança do paciente é imperativa e pode representar implicações para todo e qualquer indivíduo. A partir de características operacionalizadas pela gestão de segurança, todos os profissionais devem assumir a responsabilidade pela assistência livre de danos, encorajando-se à identificação, notificação e resolução dos problemas, cultura que, a partir da ocorrência de incidentes, promove o aprendizado organizacional e proporciona recursos, estrutura e responsabilização para a sua efetiva manutenção. ${ }^{2,5} \mathrm{~A}$ partir das metas internacionais de segurança do paciente preconizadas pela Joint Commission International $(\mathrm{JCl})$, as instituições de saúde devem buscar estratégias para trabalhar a sua implementação e identificar a ocorrência de eventos adversos, bem como reduzir ou eliminar falhas nos processos assistenciais. ${ }^{6-9}$

O processo de trabalho em saúde caracteriza-se pela sua complexidade, sendo que as atividades essenciais do enfermeiro são de caráter assistencial, gerencial, educativo, de pesquisa e de participação. ${ }^{10} \mathrm{Na}$ prática de Enfermagem, o profissional está habilitado a desenvolver a Sistematização da Assistência de Enfermagem (SAE), a qual visa a melhorar o cuidado prestado ao paciente. ${ }^{11}$ Implementada por enfermeiros, a SAE proporciona uma assistência segura e com qualidade, buscando uma melhora na comunicação e trazendo benefícios ao paciente e à equipe de saúde. ${ }^{11}$

Algumas ferramentas criadas e utilizadas na indústria, como a Análise de Modo e Efeitos de Falha, conhecida em inglês como Failure Mode and Effects Analysis (FMEA), foram direcionadas para a saúde com a finalidade de mapear, avaliar e propor 0 controle dos eventos adversos antes que ocorram. ${ }^{12} \mathrm{~A}$ Análise do Modo e do Efeito de Falhas na Assistência à Saúde, Healthcare Failure Mode and Effects Analysis (HFMEA), também é um método sistemático e proativo para avaliar processos, identificando, além das falhas, seu impacto na assistência, podendo elencar prioridades para um plano de ação. Dessa forma, a redução de erros pode trazer benefícios de uma assistência de qualidade

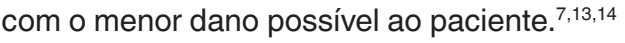

Há evidências, na literatura, que expõem a aplicabilidade das ferramentas no cotidiano assistencial em saúde. ${ }^{15-18}$ Observam-se resultados quanto à funcionalidade da sua utilização por meio do mapeamento dos eventos geradores de erros, levando à delimitação de estratégias que evitem a manifestação destes. ${ }^{15-18} \mathrm{Em}$ vista da necessidade de se aplicar metodologias para a análise do modo e do efeito de falhas na assistência à saúde, prevenindo erros e promovendo a assistência segura e de qualidade, denota-se a importância do conhecimento dos modelos de análises de falhas para a sua aplicação por profissionais de saúde e gestores como estratégia da gestão em saúde. Objetiva-se, neste artigo, discutir acerca da utilização das ferramentas de Análise de Modo e Efeitos de Falha e sua aplicação na assistência à saúde.

\section{MÉTODO}

Trata-se de um artigo de reflexão que propõe uma discussão acerca da avaliação de processos em cuidados de saúde com a aplicação das ferramentas FMEA e HFMEA, visando à segurança dos pacientes, apresentando ambos os modelos quanto às suas características e diferenças de execução.

\section{RESULTADOS}

\section{Análise de Modo e Efeitos de Falha}

A FMEA é uma ferramenta que possibilita a tomada de decisões para impedir a manifestação de erros evitáveis e observados durante a análise das etapas de um trabalho. ${ }^{1}$ É definida como uma metodologia qualitativa que permite analisar, no fluxo de trabalho, as falhas, suas causas e efeitos, levando à reflexão das ações que possibilitam a correção precoce destes erros. ${ }^{19}$

OFMEA divide-se em sete etapas: 1) selecionar um processo de trabalho a ser avaliado;2) recrutar uma equipe multidisciplinar para a aplicação; 3) estabelecer um fluxo de trabalho por parte da equipe; 4) levantar as falhas inerentes aos processos, causas e efeitos; 5) para cada falha, calcular o Índice de Prioridade de Risco (Risc Priority Number - RPN); 6) avaliar as falhas com maior RPN e definir as ações práticas e 7) recalcular o RPN após a implementação das ações de melhoria. ${ }^{20,21}$

Para selecionar um processo de trabalho, deve-se priorizar aquele que se destaca pela maior observância de falhas. Deverão ser avaliados os subprocessos para filtrar, de forma mais precisa, todas as falhas, ${ }^{20}$ após elencar uma equipe multidisciplinar, conhecedora do processo, para que identifique as causas que possam acarretar risco ou danos, priorizando as medidas corretivas. ${ }^{20,22,23}$

A equipe deverá ter um líder, o qual apresentará a ferramenta e orientará os demais membros, e um expert na ferramenta para esclarecimentos. ${ }^{16,20}$ Todos poderão alterar as etapas do diagrama de trabalho conforme a necessidade. Em seguida, para cada etapa do processo, deverão ser elencadas as principais falhas e, para cada uma, identificadas quais as suas causas e efeitos. Verificam-se, individualmente, para cada falha, utilizando-se uma escala de zero a dez, o valor de severidade (S), a ocorrência (O) e a probabilidade de detecção (D), multiplicando os valores para obter o RPN: quanto mais alto o valor atribuído à severidade e à ocorrência, e baixo para a probabilidade de detecção, maior serão o RPN e a magnitude da falha. ${ }^{19}$

De acordo com a finalidade e a característica do processo, os valores de referência da escala e seus significados poderão alterarse, porém, recomenda-se utilizar as escalas originais. ${ }^{24}$ Os pontos de corte para o RPN são determinados pelos membros de modo que os esforços se concentrem nas falhas mais importantes. ${ }^{16} \mathrm{Na}$ 
etapa seguinte, a equipe deverá atentar para a falha com maior RPN e, consequentemente, determinar ações corretivas de curto prazo. ${ }^{21}$ Todas as etapas do FMEA deverão ser documentadas, de preferência, pelo líder, para o registro de todas as falhas e os processos destacados. ${ }^{20}$ O FMEA possibilita que os responsáveis pelas práticas corretivas e seus prazos sejam registrados. ${ }^{21,25}$ Orienta-se que, após incorporadas as práticas no processo de trabalho, os membros se reúnam para recalcular o RPN e verificar se a falha foi minimizada ou eliminada. ${ }^{19}$

Sua eficácia tem sido criticada por apresentar baixa precisão. ${ }^{26}$ Entretanto, é bastante utilizada em vários cenários, principalmente na saúde, sendo reconhecida como método para aprimorar a qualidade, mesmo em situações potencialmente errôneas. ${ }^{26,27}$

\section{Análise do Modo e do Efeito de Falhas na Assistência à Saúde}

A HFMEA foi desenvolvida como uma adaptação do FMEA objetivando a análise dos pontos críticos dos serviços de saúde. ${ }^{24}$ O HFMEA também promove as ações corretivas antes que o evento adverso ocorra, sendo um modelo de análise prospectiva híbrida, pois, além de identificar o risco proativo, também faz uma análise de causa raiz. Sua aplicação concentra-se em cinco passos: 1) definir o escopo e o processo a ser analisado; 2) organizar e reunir uma equipe multidisciplinar; 3) descrever as etapas do processo em diagrama; 4) determinar o Hazard Score Matrix para cada modo de falha e 5) elaborar as ações práticas. ${ }^{23,28}$

Os participantes, conhecedores do processo em análise, deverão entrar em consenso sobre a atividade elencada para a avaliação, além de incluir a participação de um entendedor do HFMEA. Poderão ser designados subprocessos de acordo com o fluxo de trabalho pré-determinado. ${ }^{23} \mathrm{Da}$ mesma forma para cada modo de falha, a equipe observa as causas inerentes nos processos do fluxo: para uma única falha, poderão existir várias causas. ${ }^{23,28} \mathrm{Em}$ seguida, determinam-se a severidade e a frequência de ocorrência dos modos de falha, verificando-se o Hazard Score. ${ }^{14}$ A classificação de severidade e seus valores na Hazard Score incluem "catastrófico" (4), "crítico" (3), "moderado"(2) e "desprezível" (1). ${ }^{14,23}$ Já as categorias de probabilidade incluem "frequente" (muitas vezes em um ano), "ocasional" (muitas vezes em dois anos), "raro" (algumas vezes entre dois e cinco anos), e "remoto" (cinco a 30 anos), adotando-se igualmente valores de quatro a um, respectivamente. ${ }^{14,23}$ Os valores de severidade e a probabilidade deverão ser multiplicados e observada a categoria de pontuação na Hazard Sore Matrix. ${ }^{14,23,28}$

Procede-se à Árvore de Decisão na qual são verificados, entre os modos de falha levantados, aqueles que possuem maior criticidade, baixa efetividade de controle e pouca detecção, ou seja, escore igual ou maior a oito. ${ }^{14,23}$ Como criticidade, entende-se qual o impacto da falha em um determinado ponto do processo, verificando se é um ponto de fraqueza que, ao ocorrer isoladamente, gera o erro e desestabiliza o fluxo de trabalho. ${ }^{14,23}$

Trata-se de um modelo de triagem onde todas as falhas deverão passar, concentrando a atenção da equipe àquela mais grave, determinando ações em curto prazo. Fazem-se as seguintes perguntas: "o risco é intolerável? É um ponto fraco? Existem procedimentos de controle inseridos no processo? A falha é facilmente detectada a ponto de não precisar ser controlada?". ${ }^{23,28}$ Se a falha não é considerada um ponto fraco crítico no processo ou, caso considerada, já existem ações de controle inserido ou, ainda, se pode ser facilmente detectada, orienta-se para interromper a análise da Árvore de Decisão. ${ }^{14,23}$ Todavia, ao observar que há um ponto crítico, prossegue-se para o próximo passo. ${ }^{14,28}$ Assim, deverão ser designadas ações práticas de correção e os responsáveis em implementá-las. ${ }^{14,28}$

\section{DISCUSSÃO}

A FMEA e HFMEA possuem o propósito comum de prevenir falhas por meio da detecção. Em ambas, o fluxo é praticamente igual em relação à estrutura e à condução de análise dos modos de falha. Nas duas, são orientados tanto o estabelecimento de como ocorre a funcionalidade do ambiente de trabalho quanto a construção de um fluxo/diagrama que determine as etapas subsequentes. ${ }^{20}$ Igualmente, deve-se constituir uma equipe multidisciplinar e estabelecer individualmente, por modo de falha, as causas inerentes. ${ }^{14,20,21}$ Apesar de o processo ser o mesmo, as categorias numéricas utilizadas diferem. ${ }^{24}$

No FMEA, após o levantamento das causas, verificam-se os efeitos da falha sobre os processos de trabalho e, então, efetua-se o cálculo do RPN, multiplicando-se os valores de severidade, ocorrência e probabilidade de detecção de cada falha determinados por escala de zero a dez. Posteriormente, elaborase a designação das ações para corrigir ou evitar a manifestação do modo de falha. ${ }^{16}$ Para o HFMEA, após o estabelecimento das causas, para cada modo de falha, categorizam-se a severidade e a ocorrência em uma pontuação de um a quatro, multiplicandose os valores para, subsequentemente, aplicar a Hazard Score Matrix. ${ }^{14,23,28}$ Apenas as falhas com valor igual ou superior a oito são classificadas como "intoleráveis" e serão submetidas à triagem na Árvore de Decisão: apenas ao representar um ponto crítico passarão para a etapa seguinte, de orientação para a ação corretiva. ${ }^{23}$ Ao contrário, por meio do FMEA, todos os modos de falha são avaliados e recebem uma ação prática, elencando prioridades.

Essa característica pode ser um ponto limite do FMEA: se a equipe não estiver bem orientada ao correto uso e fim da ferramenta, poderá concentrar-se em vários modos de falha, possibilitando o desvio de atenção às falhas menos preocupantes. Por outro lado, as falhas classificadas como baixas ou não prioritárias podem gerar intervenções e melhorias, mesmo que pequenas, que poderão ser implementadas posteriormente.

O HFMEA, ao contrário, devido à estrutura diferenciada de triagem, a todo o momento, conduz a equipe a direcionar os esforços ao modo de falha mais grave para, então, repensar as práticas com a finalidade de impedir a ocorrência do evento adverso. Ainda sobre o cálculo do RPN na FMEA, podem ocorrer equívocos devido ao significado inversamente proporcional das categorias de classificação para a probabilidade de detecção e ocorrência; diferentes pontuações podem originar o mesmo RPN 
com o risco de não expressar a magnitude da falha. ${ }^{19,22}$ Dessa forma, um dos membros deverá ser conhecedor da ferramenta de escolha a fim de conduzir os demais para a correta utilização e esclarecimentos. ${ }^{20,25}$

Estudos mais recentes demonstraram a efetividade das ferramentas para avaliar os processos de trabalho e levantar os erros que possam prejudicar diretamente a segurança do paciente..$^{16-18}$ Há também evidências da utilização da FMEA em Centro Cirúrgico (CC) por meio da verificação de pontos críticos da assistência da Enfermagem possíveis geradores de erros. ${ }^{17,18}$ No CC, verificaram-se modos de falha com altos Índices de Riscos nas etapas de agendamento de cirurgias, distribuição de medicamentos pela farmácia satélite, preparo de materiais no Centro de Materiais e Esterilização (CME) e atividades assistenciais em CC. ${ }^{18}$ Após a aplicação das ações corretivas, evidenciou-se a queda no recálculo do índice de Risco para as quatro etapas. ${ }^{18}$

Outro estudo internacional aplicou a HFMEA para avaliar a qualidade nos processos de transfusão de sangue em uma emergência pediátrica. ${ }^{28}$ Foram identificados, ao todo, 77 modos de falha, sendo 13 identificados como risco não aceitável (Hazard Score superior a oito). Para cada um dos modos de falha, sugeriram-se ações corretivas, tais como treinamento com as equipes sobre o procedimento de transfusão de sangue, alertas para a correta identificação dos pacientes e auditorias mensais para a reavaliação dos processos. ${ }^{28} \mathrm{Em}$ ambas as ferramentas, há a possibilidade de avaliar os resultados e observar sua influência no trabalho em saúde. Os instrumentos HFMEA e FMEA demandam tempo e pleno comprometimento da equipe. ${ }^{16}$ Assim, todos os participantes devem entender a importância de analisar cada etapa e estar comprometidos para o sucesso da aplicabilidade destes instrumentos. ${ }^{16,24}$

Por meio da aplicação entre equipes, cada integrante analisa e observa os modos de falha que colocam em risco a atividade assistencial. Assim, a sua metodologia possibilita a ação de reflexão coletiva acerca da efetividade do cuidado quanto à segurança do paciente e à sugestão de ações corretivas por parte dos integrantes..$^{15} \mathrm{O}$ construir coletivo insere os profissionais como atuantes diretos de mudanças no ambiente de trabalho, possibilitando a construção de uma cultura de segurança.

\section{CONCLUSÕES E IMPLICAÇÕES PARA A PRÁTICA}

Foi possível apresentar as ferramentas FMEA e HFMEA quanto à sua utilização nos processos de saúde para a aplicação na prática, pois são métodos que auxiliam, na área da saúde, a correção de falhas antes da sua ocorrência e podem promover uma assistência segura e uma avaliação proativa de riscos relacionados ao cuidado.

Apesar da finalidade em comum, há diferenças quanto ao seguimento de utilização e cabe às equipes delimitar quais dos modelos aplicar de acordo com as características e demandas do processo de trabalho a ser analisado. Entre eles, existem distinções quanto à priorização das falhas para elencar ações práticas corretivas, principalmente no cálculo do Índice de Prioridade de Risco relacionado à gravidade e na probabilidade de ocorrência e detecção das falhas. Para tanto, os serviços devem apropriar-se dos métodos de aplicação de ambas as ferramentas e os participantes precisam ser detentores de conhecimento acerca dos processos de saúde para a garantia da efetividade $\mathrm{e} o$ alcance dos objetivos.

As instituições de saúde preocupam-se, mais intensamente nos últimos anos, com a organização da assistência isenta de falhas. Para tanto, aplicar ferramentas que avaliem os processos de trabalho, com a finalidade de alcançar o cuidado seguro, deve tornar-se uma prática nas instituições, instaurada por gestores e enfermeiros, permitindo o aperfeiçoamento dos processos de trabalho. Ao pensar em segurança do paciente na área da saúde, o FMEA e o HFMEA apontam, no sistema, erros ou a potencial ocorrência de falhas, as quais poderão manifestar-se em eventos adversos graves ao paciente. Dessa forma, mostram-se como importantes aliados para os serviços de saúde, permitindo a reflexão e a prática dos gerenciamentos de riscos e a eliminação dos eventos adversos responsáveis por reduzir o cuidado seguro ao paciente.

Proativamente, além de delimitar as falhas, possibilitam que as equipes repensem os processos, bem como as novas práticas de prevenção e se estas estão sendo efetivas ou não. Por meio da prevenção dos eventos adversos, pode-se evitar o aumento dos custos hospitalares e os riscos para os profissionais de saúde.

Portanto, as implicações deste estudo para a prática de Enfermagem destinam-se à aplicação dessas ferramentas na análise dos processos de trabalhos, no âmbito assistencial e de pesquisa, assim como considerando o enfermeiro com papel educador frente à equipe de saúde, promovendo a identificação de falhas, propondo ações de melhorias relacionadas à segurança do paciente e aproximando a prática da teoria na busca da qualidade e segurança na assistência.

Identifica-se, como limitações do estudo, que ainda há poucas pesquisas que aplicam essas ferramentas nas instituições de saúde. Entende-se que são necessários estudos futuros para a abordagem dessa temática, pois são modelos de análise de falhas com tamanho potencial para prevenir e corrigir eventos graves presentes na assistência à saúde.

\section{CONTRIBUIÇÕES DOS AUTORES}

Concepção do desenho de reflexão. Marielli Trevisan Jost. Aline Branco. Bárbara Rodrigues Araujo. Karin Viegas. Rita Catalina Aquino Caregnato.

Levantamento do referencial teórico para a condução da reflexão. Marielli Trevisan Jost. Aline Branco. Bárbara Rodrigues Araujo. Karin Viegas. Rita Catalina Aquino Caregnato.

Análise do referencial teórico. Marielli Trevisan Jost. Aline Branco. Bárbara Rodrigues Araujo. Karin Viegas. Rita Catalina Aquino Caregnato.

Interpretação do referencial teórico. Marielli Trevisan Jost. Aline Branco. Bárbara Rodrigues Araujo. Karin Viegas. Rita Catalina Aquino Caregnato. 
Redação e revisão crítica do manuscrito. Marielli Trevisan Jost. Aline Branco, Bárbara Rodrigues Araujo. Karin Viegas. Rita Catalina Aquino Caregnato.

Aprovação da versão final. Marielli Trevisan Jost. Aline Branco. Bárbara Rodrigues Araujo. Karin Viegas. Rita Catalina Aquino Caregnato.

Responsabilidade pelo conteúdo intelectual, acurácia e integridade de quaisquer partes do artigo. Marielli Trevisan Jost. Aline Branco. Bárbara Rodrigues Araujo. Karin Viegas, Rita Catalina Aquino Caregnato.

\section{EDITOR ASSOCIADO}

\section{Aline Cristiane Cavicchioli Okido}

\section{REFERÊNCIAS}

1. Lee SH, Phan PH, Dorman T, Weaver SJ, Pronovost PJ. Handoffs, safety culture, and practices: evidence from the hospital survey on patient safety culture. BMC Health Serv Res. 2016;16(1):254. http:// dx.doi.org/10.1186/s12913-016-1502-7. PMid:27405226.

2. National Patient Safety Foundation. Free from harm: accelerating patient safety improvement fifteen years after to err is human [Internet]. Boston: NPSF; 2015 [citado 26 maio 2020]. Disponível em: https://cdn.ymaws. com/www.npsf.org/resource/resmgr/PDF/Freefromharm_StateofPS.pdf

3. Farup PG. Are measurements of patient safety culture and adverse events valid and reliable? Results from a cross sectional study. BMC Health Serv Res. 2015;15(1):186. http://dx.doi.org/10.1186/s12913015-0852-x. PMid:25934272.

4. Sorra J, Gray L, Streagle S, Famarolo T, Yount N, Behm J. AHRQ hospital survey on patient safety culture: user's guide [Internet]. Rockville: Agency for Healthcare Research and Quality; 2016. (AHRQ Publication; no. 15(16)-0049-EF) [citado 26 maio 2020]. Disponível em: https://www. ahrq.gov/sites/default/files/wysiwyg/professionals/quality-patient-safety/ patientsafetyculture/hospital/userguide/hospcult.pdf

5. Brandão MGSA, Brito OD, Barros LM. Risk management and patient safety: mapping the risk of adverse events in the emergency of a teaching hospital. Rev Adm Saúde [Internet]. 2018; [citado 2020 out 24];18(70):1-13. Disponível em: http://www.cqh.org.br/ojs-2.4.8/index. $\mathrm{php} / \mathrm{ras} /$ article/view/84/125

6. Joint Commission International. International patient safety goals [Internet]. Oak Brook: Joint Commission International; 2020 [citado 20 maio 2020]. Disponível em: https://www.jointcommissioninternational. org/improve/international-patient-safety-goals/

7. Galdino SV, Reis EMB, Santos CB, Soares FP, Lima FS, Caldas $J G$ et al. Quality tools in management of health services: literature review integrative. Rev Elet Gest Saúde [Internet]. 2016; [citado 2020 out 24];7(1):1023-57. Disponível em: https://periodicos.unb.br/index. $\mathrm{php} / \mathrm{rgs} /$ article/view/3569

8. Shah A, Course S. Building the business case for quality improvement: a framework for evaluating return on investment. Future Healthc J. 2018;5(2):132-7. http://dx.doi.org/10.7861/futurehosp.5-2-132. PMid:31098548.

9. Siman AG, Brito MJM. Changes in nursing practice to improve patient safety. Rev Gaúcha Enferm. 2016;37(spe):1-9. PMid:28489153.

10. Matos DAR, Silva SOP, Lima CR. Nursing of work: addressing competencies and skills for the nurses' performance. Temas em Saúde [Internet]. 2017; [citado 2020 out 24];17(3):204-2016. Disponível em: http://temasemsaude.com/wp-content/uploads/2017/10/17314.pdf

11. Carvalho IM, Ferreira DKS, Nelson ARC, Duarte FHS, Prado NCC, Silva RAR. Systematization of nursing care in mediate post-operative of cardiac surgery. Rev Pesqui. 2016;8(4):5062-7. http://dx.doi.org/10.9789/21755361.2016.v8i4.5062-5067.

12. Stamatis DH. The ASQ Pocket Guide to Failure Mode and Effect Analysis (FMEA). Milwaukee: ASQ Quality Press; 2015.
13. Moraes CD, Rabin EG, Viegas K. Assessment of the care process with orthotics, prosthetics and special materials. Rev Bras Enferm. 2018;71(3):1099-105. http://dx.doi.org/10.1590/0034-7167-2017-0031. PMid:29924168.

14. Biazoto B, Tokarski M. Comparação entre métodos de priorização de riscos em radioterapia. Rev Bras Física Médica. 2016;10(1):17-21. http://dx.doi.org/10.29384/rbfm.2016.v10.n1.p17-21.

15. Sorrentino P. Use of failure mode and effects analysis to improve Emergency Department Handoff Processes. Clin Nurse Spec. 2016;30(1):28-37. http://dx.doi.org/10.1097/NUR.0000000000000169. PMid:26626745.

16. Hinrichsen SL, Brayner KAC, Paixão SLL, Vilella TAS, Lemos MC, Silva ED. Perception of the nursing team about causes of near miss in the medicament delay using Failure Mode and Effects Analysis - FMEA Rev Adm Saúde [Internet]. 2017; [citado 2020 out 24];17(66):1-18. Disponivel em: https://cqh.org.br/ojs-2.4.8/index.php/ras/article/view/4/15

17. Moraes CS, Rabin EG, Viégas K. Assessment of the care process with orthotics, prosthetics and special materials. Rev Bras Enferm. 2018;71(3):1099-105. http://dx.doi.org/10.1590/0034-7167-2017-0031. PMid:29924168.

18. Jost MT, Branco A, Viegas K, Caregnato RCA. Systematization of perioperatory nursing assistance: evaluating work processes in the transoperatory. Enferm Foco [Internet]. 2019; [citado 2020 out 24];10(7):43-9. Disponível em: http://revista.cofen.gov.br/index.php/ enfermagem/article/view/2354/548

19. Paranhos MM, Bachega SJ, Tavares DM, Calife NFS. Aplicação da análise de modo e efeitos de falha para o gerenciamento de riscos de um projeto. Rev S\&G [Internet]. 2017; [citado 2020 out 24];11(2016):44454. Disponível em: http://www.revistasg.uff.br/index.php/sg/article/ view/1150/551

20. Institute for Healthcare Improvement. QI essentials Toolkit: Failure Modes and Effects Analysis (FMEA) IHI Toolkit [Internet]. Boston: IHI 2017 [citado 2020 maio 26]. Disponível em: http://www.ihi.org/resources/ Pages/Tools/FailureModesandEffectsAnalysisTool.aspx

21. Ofek F, Magnezi R, Kurzweil Y, Gazit I, Berkovitch S, Tal O. Introducing a change in hospital policy using FMEA methodology as a tool to reduce patient hazards. Isr J Health Policy Res. 2016;5(30):30. http://dx.doi. org/10.1186/s13584-016-0090-7. PMid:27822358.

22. Bhattacharjee P, Dey V, Mandal UK. Risk assessment by failure mode and effects analysis (FMEA) using an interval number based logistic regression model. Saf Sci. 2020;132:1-10. http://dx.doi.org/10.1016/j. ssci.2020.104967.

23. Joint Commission Resources, Joint Commission International. Failure mode and effects analysis in health care: proactive risk reduction [Internet]. 3rd ed. Illinois: JCR; 2010 [citado 2020 maio 26]. Disponível em: https://psnet.ahrq.gov/issue/failure-mode-and-effects-analysishealth-care-proactive-risk-reduction-third-edition

24. Rah JE, Manger RP, Yock AD, Kim GY. A comparison of two prospective risk analysis methods: Traditional FMEA and a modified healthcare FMEA Med Phys. 2016;43(12):6347-53. http://dx.doi.org/10.1118/1.4966129. PMid:27908165.

25. Goodrum L, Varkey P. Prevention is better: the case of the underutilized failure mode effect analysis in patient safety. Isr J Health Policy Res. 2017;6(10):10. http://dx.doi.org/10.1186/s13584-016-0131-2. PMid:28239449.

26. Chuang S, Liu D, Wang C, Lee T. Application deficiencies of proactive analysis method for health care risk management. J Healthc Qual [Internet]. 2020; [citado 2020 out 24];14(2):34-41. Disponível em: https:// www.researchgate.net/publication/339999445_Application_deficiencies_ of_proactive_analysis_method_for_health_care_risk_management

27. Liu $\mathrm{H}$, Zhang L, Ping Y, Wang L. Failure mode and effects analysis for proactive healthcare risk evaluation: a systematic literature review. J Eval Clin Pract. 2020;26(4):1320-37. http://dx.doi.org/10.1111/jep.13317. PMid:31849153.

28. Dehnavieh R, Ebrahimipour $\mathrm{H}$, Molavi-TaleghaniY, Vafaee-Najar A, Noor Hekmat S, Esmailzdeh H. Proactive risk assessment of blood transfusion process, in pediatric emergency, using the Health Care Failure Mode and Effects Analysis (HFMEA). Glob J Health Sci. 2014;7(1):322-31. PMid:25560332. 